\title{
Datos abiertos en instituciones culturales
}

Isabel Medrano Corrales | jefa de la Unidad de Archivo y documentación de la Agencia de Obra Pública de la Junta de Andalucía

URL de la contribución <www.iaph.es/revistaph/index.php/revistaph/article/view/3978>

El texto que inició el debate sobre datos abiertos en instituciones culturales pretendía promover el intercambio de saberes en torno a cómo se experimenta el fenómeno de los datos abiertos en el ámbito cultural y generar un espacio donde compartir experiencias que se estén llevando a cabo o estén en proyecto. Aunque el enunciado del debate se refiera a instituciones culturales, tanto el texto inicial como las intervenciones se centran en un tipo de instituciones culturales: las instituciones de patrimonio histórico, que según la ley de Patrimonio Histórico Andaluz son los archivos, bibliotecas, centros de documentación, los museos y los espacios culturales (LEY 14/2007). Es por ello que para ser más precisos, hablaremos a partir de ahora de instituciones patrimoniales. A partir de las trece contribuciones al debate, se expondrán algunas de las ideas que han enriquecido la reflexión colectiva.

La función de difusión es esencial en las instituciones patrimoniales y se fortalece en proporción a la calidad democrática de cada sociedad. El cambio tecnológico que supuso Internet amplió extraordinariamente el horizonte de la difusión: catálogos en línea, exposiciones virtuales, vídeos didácticos, visitas virtuales, etc. Abrir datos de archivos, bibliotecas y museos es un nuevo hito en esta evolución alentada por varios factores: atender las demandas actualizadas de una ciudadanía cada vez más conocedora y consciente, mejorar el nivel de reputación de la administración pública afectada por escándalos de corrupción y promover que la información pública sea materia prima en la economía del conocimiento y revierta en beneficio del desarrollo económico y el empleo. Como dice Julián Moyano, "no es ningún fin en sí mismo; es un medio que va a posibilitar nuevos recursos y servicios gracias a la reutilización de estos".

Existe consenso al entender la transparencia como línea fundamental de inspiración y actuación del Gobierno
Abierto $^{1}$. En este contexto, los datos públicos han de estar disponibles en la web para que cualquier ciudadano acceda a ellos sin ninguna restricción. Sin embargo, una visión estrictamente tecnocentrista nos llevaría a un resultado incompleto. Un proyecto de datos abiertos encuentra su fundamento en el desarrollo histórico de las sociedades democráticas y viene a favorecer el rendimiento de cuentas de las acciones del Estado, así como reducir la brecha entre representados y representantes (PRINCE; JOLIAS, 2013). Por tanto, como nos sugiere en su contribución Eréndira Sarahí Pérez, debe aspirar a convertirse en una plataforma no sólo de difusión, sino también de confluencia con infomediarios y la ciudadanía en general para co-participar en la generación y retroalimentación de información pública. Todo ello con la finalidad de fomentar la reutilización de datos públicos para generar nuevos productos y servicios, así como promover una ciudadanía más informada y consciente.

La apertura de información es tendencia en la gestión administrativa y económica de las agendas gubernamentales a nivel internacional desde la publicación de The 8 Principles of Open Government Data ${ }^{2}$ en 2007, el Memorandum de la Open Government Initiative firmado por el presidente Obama en 2009, así como la promoción de la Alianza para el Gobierno Abierto (AGA) de 2011, más conocida como Open Government Partnership.

La OCDE, organización activa en la defensa de los datos públicos abiertos, en la cumbre ministerial de Economía Digital, celebrada en Cancún en junio de 2016, en la que participaron 43 países, coincidió en afirmar que la digitalización puede ser la clave para impulsar un mejor desarrollo económico, más justo, sostenible e inclusivo.

En 2010 la Comisión Europea diseñó la Agenda Digital para Europa como una de las siete iniciativas emblemá- 
ticas de la estrategia Europa 2020. En 2017 ha presentado la revisión de dicha estrategia, apuntando tres nuevos ámbitos de actuación que requerirán una atención especial para conseguir un entorno digital más equitativo, abierto y seguro: (1) la promoción de las plataformas en línea, (2) el desarrollo de la economía de los datos europea (3) y la promoción de un ecosistema cibernético fiable.

En España, la nueva Estrategia Digital para una España Inteligente -que se encuentra actualmente en consulta pública (CONSULTA, 2017) - se articula en torno a cinco pilares: (1) economía de los datos, (2) ecosistemas 4.0, (3) regulación inteligente, (4) infraestructuras tecnológicas y (5) ciudadanía y empleo digital. En esta nueva estrategia digital los datos pasan así a ocupar un lugar de máxima relevancia, en lo que ya se empieza a denominar como economía de los datos.

En su contribución al debate, Noemí Lera propone una respuesta a la pregunta ¿por qué los datos abiertos? porque favorecen la circulación de información hacia los agentes económicos y la ciudadanía, porque los datos abiertos constituyen una materia prima para crear productos, servicios y conocimiento y porque los datos abiertos generan tejido empresarial y productivo. A estas razones, desde el Grupo Datasea añaden que los datos abiertos estimulan la innovación, promueven descubrimientos adicionales y mejoran avances científicos más económicos. Otros autores ponen más el énfasis en los beneficios del acceso a la información pública para la construcción de una democracia con un reparto de poderes más equitativo (FARREL, 2012).

Ciertamente una inmensa cantidad de sucesos y eventos que nunca antes pudieron medirse, almacenarse, analizarse y compartirse están convirtiéndose ahora en datos. Se trata de información procedente de los sensores inalámbricos, los dispositivos móviles, los históricos de aplicaciones (por ejemplo los logs), las cámaras (sistemas de teledetección), micrófonos, lectores de identificación por radiofrecuencia, etc. La mejora de las tecnologías para recopilar y tratar estos datos masivos supone un paso importante en el esfuerzo por comprender el mundo.

Las instituciones patrimoniales, que en un primer momento quedaron excluidas del cumplimiento de la Ley de reutilización de información del sector público (RISP), hace dos años fueron incorporadas, a raíz de la trasposición de la DIRECTIVA 2013/37/UE. Este cambio legal responde al importante volumen de recursos de información que poseen las instituciones de patrimonio y los proyectos de digitalización que vienen llevando a cabo (LEY 18/2015). Esta situación de incorporación paulatina se extrapola a otros países, como Argentina y México, según nos informan en sendas contribuciones al debate.

El mandato de la reutilización de la información pública de instituciones patrimoniales implica también el cumplimiento de la Norma Técnica de Interoperabilidad de reutilización de recursos de la información, que establece las condiciones para facilitar y garantizar el proceso de reutilización de la información pública, asegurando su persistencia, el uso de formatos, así como los términos y condiciones de uso adecuados (RESOLUCIÓN, 2013).

Como advierte Noemí Lera, el ámbito de la cultura tiene una composición institucional compleja: por un lado, instituciones, organismos y entidades públicas; y por otro, entidades y fundaciones privadas, algunas de las cuales reciben fondos públicos. Cada una tendrá que abordar su política de datos abiertos según la normativa aplicable en cada caso, así como las directrices de política digital globales de cada administración o entidad. Esta fragmentación competencial puede proyectar también una fragmentación en el panorama de la apertura de datos culturales. La solución pasa por una mayor cooperación y coordinación en la apertura de los datos por parte de las instituciones implicadas, asegurando así la interoperabilidad no solo técnica, semántica y legal, sino también organizativa (DESCENTRALIZACIÓN, 2017).

Las instituciones patrimoniales públicas en España, en general, optan por dos vías para cumplir con el man- 


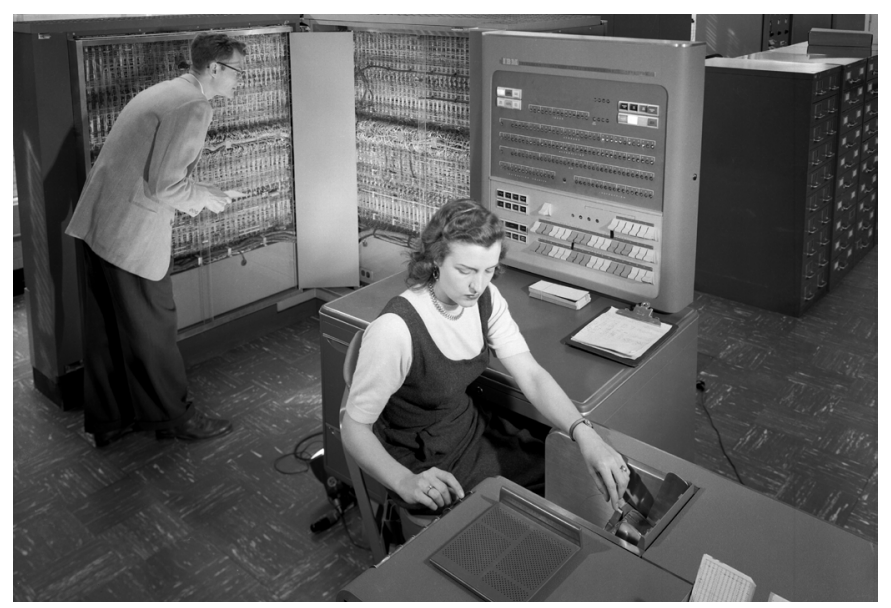

Máquina de Datos Electrónicos IBM | foto NASA on The Commons

dato de la Ley de reutilización de información del sector público, de difundir "los documentos producidos o conservados" en archivos, bibliotecas y museos -esta publicación, recordemos, debe cumplir con los requisitos de los datos abiertos-. La primera sería la publicación de los datos abiertos en plataformas con infraestructura propia de la institución. Sería el caso de: datos.bne.es de la Biblioteca Nacional de España; la Lista de Encabezamientos para Bibliotecas Públicas en SKOS, de la Subdirección General de Coordinación Bibliotecaria; los Tesauros del Patrimonio Cultural de España en Linked Open Data publicados por la Subdirección General de los Museos Estatales.

La segunda vía sería la publicación de los conjuntos de datos culturales en portales transversales, ya sean los portales de datos abiertos de cada administración -por ejemplo, el portal de datos abiertos del Estado, el portal de datos abiertos del Ayuntamiento de Madrid, etc.-, o bien otros especializados en una materia: cartografía, estadística, investigación, etc. Este sería el caso del portal de archivos aragoneses DARA, que ofrece los registros descriptivos en formatos abiertos a través del portal Aragón Open Data. Estas vías no son excluyentes en un doble sentido: una institución puede optar por una u otra en función de las características de cada conjunto de datos; $y$, por otra parte, puede simultáneamente

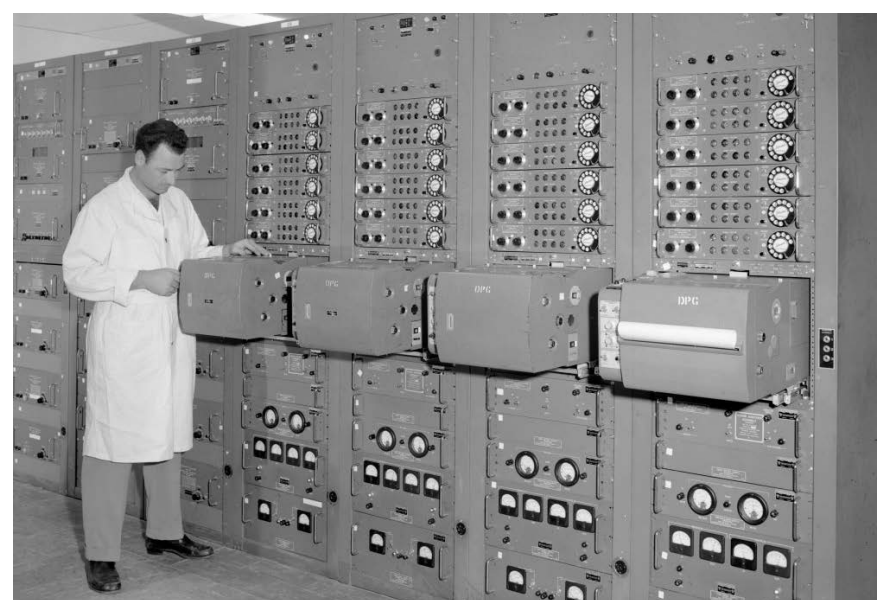

Paneles de Control. Equipo de procesamiento de datos | foto SDASM Archives

publicar en su propia plataforma y en un portal general de datos abiertos. En este sentido, es paradigmático el caso estadounidense de la Library of Congress, que recientemente ha publicado 25 millones de registros bibliográficos en su catálogo en línea y en el portal de datos abiertos gubernamental.

En el catálogo de Datos Abiertos del Estado hay más de 15.000 conjuntos de datos, de los que solo el $6 \%$ está catalogado como datos relativos a la cultura y el ocio. Esta falta de tipificación se repite en otros catálogos de datos, lo cual supone un problema generalizado para las búsquedas y análisis de datos -este problema es puesto de manifiesto en el debate por el Grupo iArtHis_Lab, para lo que recomiendan realizar taxonomías de clasificación más rigurosas-. La Administración General del Estado y sus organismos vinculados o dependientes son los únicos obligados a publicar en este catálogo. El resto de administraciones pueden hacerlo de forma opcional y complementaria a la publicación en sus propios catálogos. La mayoría de los conjuntos de datos culturales provienen de comunidades autónomas y ayuntamientos. Y son muy pocas, como comenta Noemí Lera, las instituciones culturales que aparecen como publicadoras de conjuntos de datos: la Biblioteca Nacional de España, el Instituto Nacional de las Artes Escénicas y de la Música y la Fundación Lázaro Galdiano. 


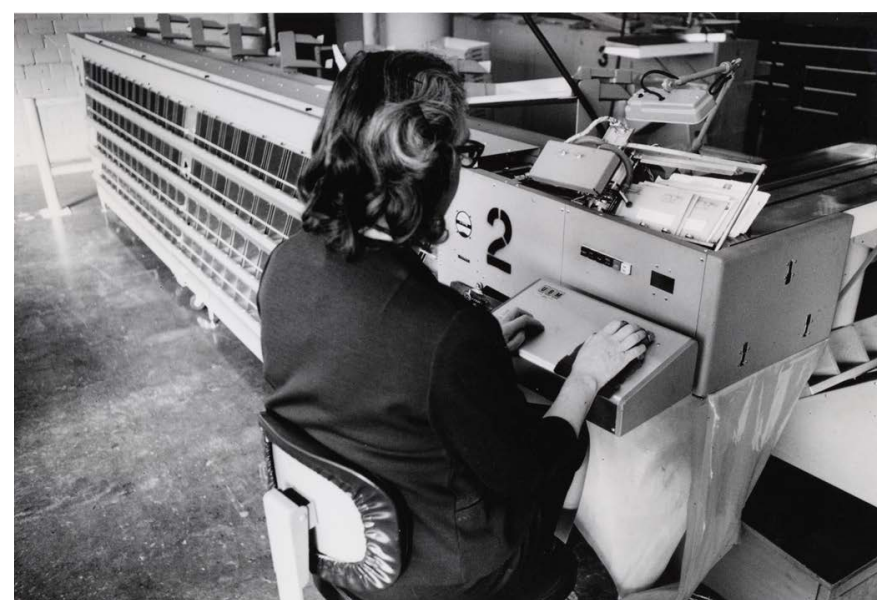

Mujer trabajando en un centro de procesamiento del correo | foto Smithsonian Institution

Es esencial que la información "liberada" sea cuantitativa y cualitativamente relevante a los fines de contribuir a la transparencia y participación; de lo contrario los datos abiertos dejarían de prestar su función en el camino al gobierno abierto. En este contexto surge el concepto de la competencia mediática de la ciudadanía, entendida como la capacidad para hacer un uso libre y crítico de aquellos datos que pueden ser utilizados de forma abierta, reutilizándose y redistribuyéndose por cualquier persona para la creación de nuevos contenidos (GURSTEIN, 2011).

En su contribución, el grupo Datasea expresa su certeza de que el futuro reside en cambiar la cultura de trabajo y uno de los vértices del éxito será la colaboración con los grupos de interés involucrados, de forma que se favorezcan las sinergias entre Administración Pública y ciudadanía. Por tanto, es fundamental que las administraciones no solo abran datos, sino también el proceso de elección de los conjuntos de datos y elementos de metadatos a publicar. La participación de los agentes implicados es esencial desde el inicio para cumplir las expectativas con éxito y que los datos abiertos no solo se publiquen, sino que sean realmente reutilizables. Se hace necesario para ello reforzar la formación en metodologías y técnicas de participación ciudadana, a los equipos implicados en los proyectos de datos abiertos.

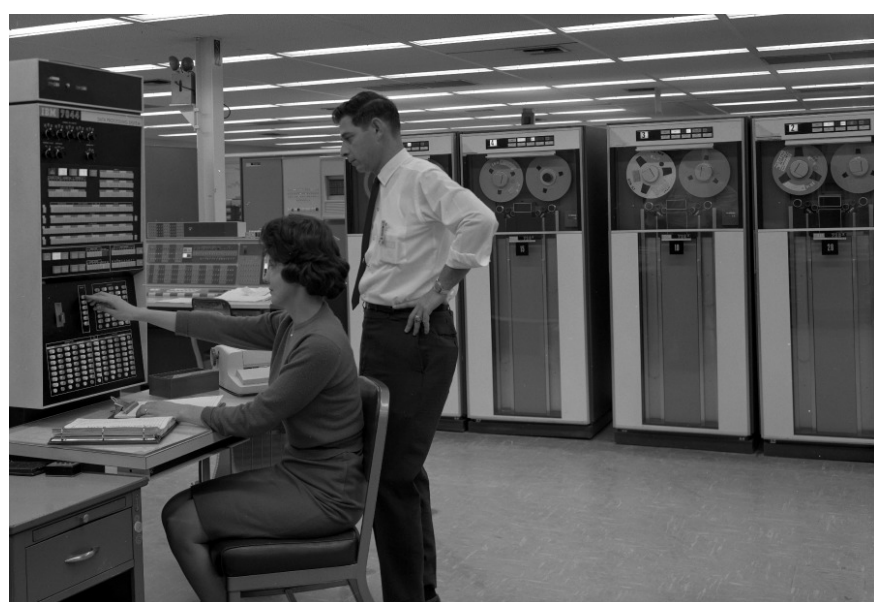

Will Herman introduciendo registros. Centro de procesamiento de datos | foto Atlas Collection Image. SDASM Archives

Partiendo de la calidad de los datos que se pueden extraer de los expedientes administrativos electrónicos y más concretamente de los expedientes de contratación, se desarrolló un proyecto piloto en el marco de un trabajo de investigación, que viene al caso comentar como experiencia de participación. Para validar la selección de los conjuntos de datos que se iban a publicar en abierto, así como fijar la prioridad para publicarlos, se aplicó la metodología de participación de agentes implicados a través de la entrevista directa. Posteriormente se promovió la interacción de todos los participantes a través de un foro de debate con interesantes resultados, que llevaron a la adaptación del perfil de metadatos para incorporar las sugerencias acordadas (MEDRANO CORRALES; BUENO DE LA FUENTE; MÉNDEZ RODRÍGUEZ, 2016).

Y llega el momento de preguntarnos ¿qué datos son los que deben publicar en abierto las instituciones patrimoniales? Como ya se ha citado, la Ley de reutilización de la información habla de "los documentos producidos o conservados" por archivos, bibliotecas y museos. Por su parte, las leyes de transparencia, para las entidades sujetas a su cumplimiento, fijan la información que debe ser objeto de publicidad activa. Ahora bien, la transparencia es ante todo una actitud que debe inspirar la transformación de las instituciones en mucho más abiertas y predispuestas a publicar importantes y numerosos con- 
a debate Datos abiertos en instituciones culturales

| coordina Isabel Medrano Corrales

juntos de datos. Evidentemente, habrán de considerarse las limitaciones de acceso a la información fijadas por la legislación, que en el caso del ámbito cultural generan muchas dudas en su aplicación. Esta cuestión parece ser global a tenor de la intervención en el debate sobre el caso mexicano, que pone de manifiesto la discusión en torno a los bancos de imágenes sobre si dar acceso restringido con imágenes a baja resolución o permitir su descarga; o la cuestión del riesgo que comporta para la conservación de determinados bienes culturales la publicación de información detallada.

La expansión de los derechos de autor hacia la reproducción de obras artísticas o piezas históricas en dominio público supone en muchos casos una interferencia en el derecho de acceso. Anabel Fernández defiende que la propiedad intelectual se refiere a las creaciones de la mente protegidas por los derechos de autor siempre que se demuestre su originalidad. Por tanto, la definición de originalidad juega un papel fundamental para solucionar las posibles dudas en las políticas de acceso a las colecciones (FERNÁNDEZ MORENO, 2016).

Partiendo de casos reales de apertura de datos de instituciones patrimoniales y haciendo un ejercicio de identificación de otros posibles, algunos de los cuales han sido propuestos en el debate, a continuación apuntaremos una serie de fuentes de información de interés para su tratamiento y publicación como datos abiertos.

Las instituciones patrimoniales han desarrollado repositorios institucionales para promover el acceso abierto a sus colecciones de recursos digitales, con una doble finalidad de preservación y difusión de los mismos. Es la solución empleada con éxito por el Laboratorio de Documentación Geométrica del Patrimonio de la Universidad del País Vasco, como se indica en el debate. A nivel estatal, Hispana es el portal de acceso a la cultura digital y el agregador nacional de contenidos a Europeana, que reúne las colecciones digitales de archivos, bibliotecas y museos españoles. Es cierto que las instituciones culturales publican cada vez más datos, un buen ejemplo son los más de 2 millones de recursos publica- dos en Europeana provenientes de más de 80 instituciones españolas. Sin embargo, en la inmensa mayoría de los casos, la reutilización de esos recursos tiene restricciones, casi siempre asociadas al uso comercial de los mismos. Estos repositorios que gestionan contenidos digitales y sus correspondientes metadatos, están muy bien posicionados para dar el salto a la publicación de datos abiertos. Ambos -objetos digitales y metadatos- son susceptibles de evolucionar y publicarse también como datos abiertos. En este punto se encuentra el Instituto Andaluz del Patrimonio Histórico, que como se ha comentado en el debate, acaba de comenzar la implantación de su proyecto Datos Abiertos del Patrimonio Cultural Andaluz.

Los instrumentos de descripción de los fondos y colecciones patrimoniales: guías, catálogos e inventarios constituyen una información muy valiosa para la investigación, la educación o la creación, que no siempre es accesible. Al igual que en el caso de los repositorios institucionales, las bases de datos descriptivas de archivos, bibliotecas y museos tienen una posición privilegiada para su transición hacia la publicación como datos abiertos cinco estrellas. En esta situación estarían, por ejemplo, los catálogos de las colecciones de museos andaluces, los catálogos de museos nacionales, el portal de archivos españoles o el catálogo colectivo de bibliotecas públicas.

Como dice Julián Moyano, la información descriptiva bibliográfica, museística y documental debe acompañar a una apertura de la propia institución, aportando cualquier tipo de información estructurada bajo formatos y condiciones de datos abiertos: la agenda de eventos, estadísticas de servicios, presupuesto, perfiles profesionales, cargos, novedades, últimas adquisiciones, donaciones, etc. Son datos de gestión de la institución, que están contenidos en las aplicaciones informáticas de tramitación o bien en los documentos administrativos. Tanto las evidencias como los documentos, en el actual entorno de gestión administrativa electrónica, deben ser tenidos en cuenta en el plan de datos abiertos de cada institución. 
Las instituciones patrimoniales tienen información cartográfica, georreferenciada y estadística relativa a los bienes culturales que gestionan, así como a los servicios que presta: mapas de situación de bienes de interés cultural, planos de bienes inmuebles, estadística de los servicios que prestan los archivos, bibliotecas y museos, etc. En el debate, Emilio J. Mascort-Albea propone la publicación en abierto de información cartográfica a escala arquitectónica, para un mayor y mejor conocimiento de los edificios patrimoniales, así como de los bienes muebles que se localicen en su interior. Por su parte, Sergio Reyes-Corredera pone de relieve el importante papel de los centros de estadística como catalizadores de los datos abiertos de instituciones patrimoniales y los de la actividad económica ligada al sector turístico.

Los datos resultado de investigación están excluidos del objeto de la Ley de reutilización de la información del sector público ${ }^{3}$. No obstante, ya se vislumbran cambios dirigidos hacia la apertura. La European University Association (EUA) es la organización representativa de universidades y conferencias de rectores nacionales en 47 países europeos, y juega un papel influyente en las políticas de la UE en materia de educación superior, investigación e innovación. En la encuesta institucional 2015/2016 que la EUA realiza a 169 instituciones de 33 países europeos, se muestra una conciencia creciente sobre el acceso abierto a los datos de la investigación (MORAIS; BORRELL-DAMIAN, 2017).

Por su parte, la Unión Europea ha anunciado que todos los trabajos científicos financiados con fondos públicos publicados en Europa podrían estar en libre acceso para el año 2020, una decisión encaminada al modelo de ciencia abierta. Esta medida afectaría a la producción científica de instituciones culturales que están reconocidas como centros de investigación, por ejemplo el Instituto Andaluz de Patrimonio Histórico.

Dos intervenciones en el debate nos ofrecen la ocasión de diferenciar los datos para la investigación y los datos de investigación. En el ámbito de los datos abiertos, los primeros son los datos públicos que sirven al proceso de investigación; y los segundos son los obtenidos en los procesos de experimentación, observación, simulación, medición, cálculo, análisis, etc., compilados en diversidad de formatos y archivos. En el proyecto de investigación Exhibitium del Grupo iArtHis_Lab de la Universidad de Málaga, para la generación de conocimiento sobre exposiciones artísticas temporales constataron la inexistencia de datos abiertos sobre exposiciones temporales de arte, cuestión que determinó el desarrollo del proyecto de investigación. Por su parte, Alfonso Monsalve manifiesta lo difícil que resulta el acceso de los investigadores a los materiales arqueológicos extraídos de excavaciones y a los datos científicos producidos durante las mismas. Considera que los datos en abierto mejorarían e incrementarían la capacidad investigadora de nuestro país y, en otra vertiente, paliarían la falta de inversión en ciencia.

Ahora bien, la función de las instituciones patrimoniales en la apertura de datos debe ir más allá de su publicación en línea, siendo importante promover la cultura de la apertura de información y fomentar su reutilización. Según la Ley 18/2015 "se entiende por reutilización el uso de documentos que obran en poder de las Administraciones y organismos del sector público, por personas físicas o jurídicas, con fines comerciales o no comerciales, siempre que dicho uso no constituya una actividad administrativa pública" (art. 3.1). Aunque la normativa no contempla el papel activo de los reutilizadores de la información, es evidente que para cumplir con éxito los objetivos de transparencia y satisfacción de las demandas informativas, es preciso contar con la participación de los demandantes/consumidores/reutilizadores de la información. Para ello sería conveniente disponer de canales de comunicación con la administración y la participación de los usuarios en órganos decisorios dentro del plan de datos abiertos (MEDRANO CORRALES; BUENO DE LA FUENTE; MÉNDEZ RODRÍGUEZ, 2016). Son muy convenientes iniciativas como la que surgió en Dinamarca en 2011 denominada Sharing is caring ("Compartir es amar"), a través de la cual se organizan seminarios que impulsan la apertura de la información del sector GLAM (galerías, bibliotecas, archivos y museos, por sus siglas en inglés). El próximo mes de noviembre se celebrará la 


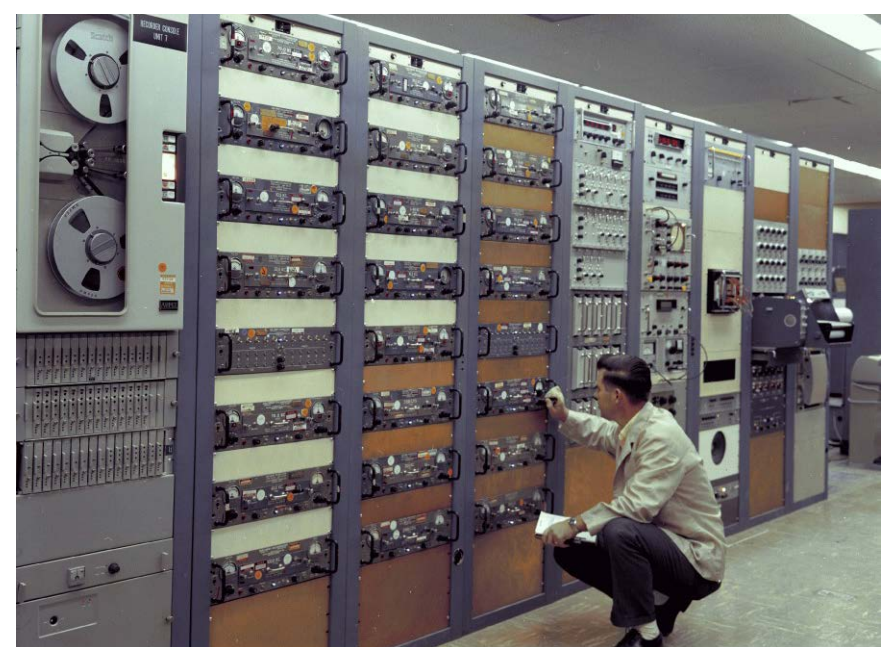

Laboratorios de Datos | foto Atlas Collection Image. SDASM Archives

quinta edición del seminario Sharing is Caring en Aarhus (Dinamarca), donde se hablará del papel que desempeña la apertura de los datos culturales en la participación ciudadana y su impacto en el conjunto de la sociedad. En Andalucía recientemente se ha puesto en marcha una iniciativa en este sentido: el Laboratorio Abierto de Patrimonio (LAP) del Instituto Andaluz del Patrimonio Histórico, que organiza actividades formativas para promover la filosofía de gobierno abierto, basada en la transparencia institucional, la colaboración y creación de redes con diferentes agentes, y la participación de la ciudadanía y los profesionales facilitando así nuevas conexiones.

El sector de actividad económica derivado de la reutilización de datos abiertos se denomina sector infomediario. El último informe elaborado por el Observatorio Nacional de las Telecomunicaciones y de la Sociedad de la Información (ONTSI) revela que el subsector cultural supone un $13 \%$ del sector infomediario (CARACTERIZACIÓN, 2016). Dicho informe contiene una valoración de los datos abiertos, en el que las empresas puntúan positivamente los datos culturales por estar en formatos estructurados, no requerir un registro previo y sobre todo, la gratuidad.

En su contribución al debate, Sergio Reyes-Corredera advierte de las complicaciones que los emprendedores se encuentran para la explotación de los datos culturales, al no disponer de datos sobre el perfil del consumidor del bien o servicio cultural, la competencia empresarial con la que se pueden encontrar, etc. Como solución reclama que se oferten datos en abierto relativos al volumen de visitantes de los espacios y eventos culturales, sus perfiles socioeconómicos, el lugar de procedencia, el interés que les hace asistir a un acto, evento o ruta cultural, entre otros parámetros.

En Francia se ha puesto en marcha el Proyecto Etalab para coordinar la política de reutilización de datos abiertos en la administración pública. Tras una consulta pública ha concluido que los datos públicos abiertos culturales gratuitos y reutilizables más interesantes son: los datos económicos y estadísticos, los metadatos de obras culturales y las imágenes y copias digitales de obras en dominio público.

Julián Moyano nos habla de la capacidad de los datos de archivos, bibliotecas y museos de aportar conocimiento y enriquecer a otros contenidos. Los datos abiertos patrimoniales fomentan el desarrollo de nuevos servicios que se sirven o apoyan en estos datos. Ponemos algunos ejemplos de reutilización: mapas enriquecidos (Biblioguía, un mapa y localizador de bibliotecas en España, en versión web y móvil, que extrae la información de los portales de datos abiertos; el Mapa interactivo de los bienes de interés cultural de Aragón, una iniciativa que están desarrollando un grupo de alumnos de tercer curso de Educación Secundaria Obligatoria de Zaragoza), aplicaciones web y móvil (AgendaOberta es un agregador de actividades de todo tipo que se programan en Cataluña, que extrae la información automáticamente de las webs institucionales básicamente), aplicaciones científicas (la propuesta comentada en el debate de un estudio de evaluación de la política pública cultural y de ocio a partir del conjunto de datos Agenda 2017 del Ayuntamiento de Málaga, mediante minería de texto).

Una de las oportunidades a destacar para las instituciones patrimoniales es la confluencia entre los proyec- 
tos de ciudades inteligentes y de datos abiertos. Es un campo interesante para explorar la participación en el desarrollo de los destinos turísticos inteligentes como una nueva vía de afianzar el papel de los archivos, bibliotecas y museos (VALENTÍN RUIZ; BENITO BLÁZQUEZ, 2017).

Podemos apreciar cómo la reutilización nos va mostrando nuevos usos del patrimonio. En palabras de la directora de la Biblioteca Nacional de España, Ana Santos Aramburo, su objetivo es "ofrecer la posibilidad de reutilizar nuestros datos y nuestros contenidos culturales para nuevos usos, además de para la investigación, para usos que hasta ahora eran insospechados como el ocio, el turismo, la moda o los videojuegos" (BNELAB, 2017).

Al margen de la reutilización, la apertura de datos tiene otras ventajas que benefician a las instituciones patrimoniales y a la comprensión de los bienes culturales. Las administraciones nunca tuvieron tal volumen de información propia organizada para analizar, extraer conclusiones y marcar nuevas estrategias. Las posibilidades para evaluar las políticas públicas culturales se van a ir ampliando progresivamente a medida que se incremente la publicación de datos abiertos. Datos que se podrán usar también para tomar decisiones técnicas sólidamente fundamentadas.

La apertura de datos de los instrumentos de descripción de las instituciones patrimoniales ofrece la posibilidad de enlazar dichos datos, generando un contexto enriquecido para cada obra y conectando a las distintas instituciones de memoria.

Eréndira Sarahí Pérez reflexiona en el debate sobre si con este proceso de apertura de datos se ha conseguido una sociedad más consciente y quiénes están siendo los verdaderos beneficiarios de la reutilización. Desconocemos si las colecciones de datos son catalizadores del progreso y contribuyen a la democratización de la educación y la ciencia y a mejorar el desarrollo económico y social y si, finalmente, estos podrán llegar a ser económicamente rentables. Como dice Noemí Lera, aun- que no existen indicadores de referencia para valorar el impacto de los datos abiertos para el ámbito cultural, la valoración debe realizarse teniendo en cuenta distintas dimensiones, como la económica, que se puede ligar al desarrollo del sector turístico, la social, por su incidencia en servicios públicos como la educación, o la tecnológica, por la capacidad de innovar.

\section{NOTAS}

1. "El concepto de Gobierno abierto (Open Government) tendría un mayor alcance que lo que entendemos por administración electrónica, de acuerdo con el autor Jesús García García (2014), estaríamos ante un nuevo modelo administrativo, más abierto y participativo, en el que los ciudadanos puedan acceder libremente a la información pública para poder opinar y valorar, permitiéndoles colaborar y tomar decisiones basadas en sus propias preferencias y necesidades" (CORONADO MARíN, 2015: 10).

2. Para que se consideren abiertos, los datos deben cumplir con los siguientes requisitos: completos, primarios, actuales, accesibles, procesables, no discriminatorios, no propietarios y sin licencia (OPEN GOVERNMENT WORKING GROUP, 2007, 2007).

3. La Ley $18 / 2015$ no es aplicable a "Los documentos producidos o conservados por instituciones educativas y de investigación (incluidas las organizaciones para la transferencia de los resultados de la investigación, centros escolares y universidades, exceptuando las bibliotecas universitarias) así como los museos y archivos estatales como agentes de ejecución del Sistema Español de Ciencia, Tecnología e Innovación siempre que sean resultado de una investigación" (art. 3.3.g). 


\section{BIBLIOGRAFÍA}

- BNELAB, reutilizar datos abiertos de la Biblioteca Nacional Española es ya una realidad (2017) En Datos.gob.es [en línea] publicada el 15 de junio de 2017 <http://datos.gob.es/es/ noticia/bnelab-reutilizar-datos-abiertos-de-la-biblioteca-nacional-espanola-es-ya-una-realidad> [Consulta: 19/09/2017]

- CARACTERIZACIÓN del Sector Infomediario en España (2016) sl: Observatorio Nacional de las Telecomunicaciones y de la Sociedad de la Información (ONTSI). Entidad Pública Empresarial Red.es. Secretaria de Estado para la Sociedad de la Información y la Agenda Digital. Ministerio de Energía, Turismo y Agenda Digital, 2016 <http://www.ontsi.red.es/ontsi/es/ content/estudio-de-caracterizaci\%C3\%B3n-del-sector-infomediario-2016> [Consulta: 03/09/2017]

- CORONADO MARÍN, V. J. (2015) La gestión de los Datos Abiertos en la Administración. Salamanca: Universidad de Salamanca, Máster en sistemas de información digital, 2015 <https://gredos.usal.es/jspui/bitstream/10366/131723/1/TFM SistemasInfoDigital CoronadoMarin VictorJose SI 60 20152016.pdf> [Consulta: 11/09/2017] Trabajo fin de Máster inédito

- CONSULTA Pública sobre la Estrategia Digital para una España Inteligente (2017) Secretaría de Estado para la Sociedad de la Información y la Agenda Digital. Ministerio de Energía, Turismo y Agenda Digital <http://www.minetad.gob.es/telecomunicaciones/es-ES/Participacion/Documents/estrategia-digital-espana-inteligente.pdf> [Consulta: 03/09/20 17]

- La DESCENTRALIZACIÓN administrativa, un desafío para el uso de los datos públicos (2017) En Datos.org.es [en línea], publicado 23 de junio de 2017 <https://datos.gob.es/ es/noticia/la-descentralizacion-administrativa-un-desafio-para-el-uso-de-datos-publicos> [Consulta: 03/09/2017]

- DIRECTIVA 2013/37/UE, de 26 de junio, por la que se modifica la Directiva 2003/98/CE relativa a la reutilización de la información en el sector público. Diario Oficial de la Unión Europea (DOUE), n. ${ }^{\circ}$ 175, de 27 de junio de 2013, pp. 1-8

- FARREL, H. (2012) Trish, Reiner and the Politics of Open Data. En Out of the crooked timber of the humanity no straight thing was ever made [en línea], publicado el 4 de julio de 2012 <http://crookedtimber.org/2012/07/04/trish-reiner-and-the-politics-of-open-data/> [Consulta: 03/09/2017]

- FERNÁNDEZ MORENO, A. (2016) Originalidad es la clave. Propiedad Intelectual, Dominio Público y el acceso a las colecciones culturales. Cuadernos de Arte de la Universidad de Granada, n. ${ }^{\circ}$ 47, 2016, pp. 131-146 <http://revistaseug.ugr.es/index php/caug/article/download/5429/5088> [Consulta: 03/09/2017]

- GURSTEIN, M. (2011) Open data: Empowering the empowered or effective data use for everyone? First Monday [en línea] vol. 16, n. ${ }^{\circ}$ 2, 7 febrero $2011<$ http://firstmonday.org/article/view/3316/2764> [Consulta: 03/09/2017]
- LEY 14/2007, 26 de noviembre, del Patrimonio Histórico de Andalucía. Boletín Oficial de la Junta de Andalucía, n. ${ }^{\circ} 248$, de 26 de noviembre de 2007, pp. 6-29

- LEY 18/2015, de 9 de julio, por la que se modifica la Ley $37 / 2007$, de 16 de noviembre, sobre reutilización de la información del sector público. Boletín Oficial del Estado, n. ${ }^{\circ} 164$, de 10 de julio de 2015, pp. 57436-57450

- MEDRANO CORRALES, I; BUENO DE LA FUENTE, G; MÉNDEZ RODRÍGUEZ, E. (2016) Participación ciudadana en la elección de datos abiertos de expedientes de contratación. Tábula, n. ${ }^{0} 19,2016$, pp. 67-84

- MORAIS, R.; BORRELL-DAMIAN, L. (2017) Open access 2015-2016 EUA Survey Results [en línea] Brussels: European University Association (EUA), junio 2017 <http://www.eua.be/ Libraries/publications-homepage-list/oa-survey-2015-2016-results> [Consulta: 03/09/2017]

- OPEN GOVERNMENT WORKING GROUP (2007) Principles of Open Government Data en 7-8 Diciembre 2007, Sebastopol, California <https://opengovdata.org/> [Consulta: 15/09/2017]

- PRINCE, A.; JOLIAS, L. (2013) Las Fuentes Conceptuales Del Gobierno Abierto. Revista TELOS (Cuadernos de Comunicación $E$ Innovación), n. ${ }^{\circ}$ enero-abril, pp. 1-9 <https://telos. fundaciontelefonica.com/url-direct/pdf-generator?tipoContenido=articuloTelos\&idContenido=2013021317570001\&idioma=es> [Consulta: 03/09/2017]

- RESOLUCIÓN de 19 de febrero de 2013, de la Secretaría de Estado de Administraciones Públicas, por la que se aprueba la Norma Técnica de Interoperabilidad de Reutilización de recursos de la información. Boletín Oficial del Estado, n. ${ }^{\circ} 54$, de 4 de marzo de 2013, pp. 17045-17071

- VALENTÍN RUIZ, F. J.; BENITO BLÁZQUEZ, B. (2017) El valor de la biblioteca en la construcción de ciudades inteligentes. En VIII Congreso Nacional de Bibliotecas Públicas. Espacio Físico y virtual (2016. Toledo). sl: Ministerio de Educación, Cultura y Deporte, Secretaría General Técnica, Subdirección General de Documentación y Publicaciones, 2016, pp. 226-233 <https://sede.educacion.gob.es/publiventa/d/20877C/19/0> [Consulta: 03/09/2017] 